\title{
Inclusion complex of Artemether with 2- hydroxypropyl- $\beta$-cyclodextrin for the treatment of malaria: preparation, characterization and evaluation
}

\begin{abstract}
Artemether (ATM) is being evaluated for use in Malaria at present. However, its poor solubility in water limits its exploitation and application in clinical. The purpose of this work is to develop inclusion complex of ATM with 2-hydroxypropyl- $\beta$ cyclodextrin (ATM-2-HP- $\beta-\mathrm{CD}$ inclusion complex) which have the ability to improve the solubility and oral bioavailability of ATM. The ATM-2-HP- $\beta-$ CD inclusion complex was prepared by Solution mixing method at the mole ratio of 1:36 (ATM: HP $-2-\beta-C D$ ). The average drug entrapment efficiency and loading capacity were $97.66 \pm 1.22 \%$ and $2.64 \pm 0.03 \%$, respectively. The formation of ATM-2-HP- $\beta$ $\mathrm{CD}$ inclusion complex was verified by differential scanning calorimetry (DSC) and Fourier transform Infrared spectroscopy (FT-IR). The solubility of ATM-2-HP- $\beta$ $\mathrm{CD}$ inclusion complex was $11.28 \pm 2.26 \mathrm{mg} / \mathrm{ml}$, having improved vastly compared to ATM of just $1.18 \mathrm{ug} / \mathrm{ml}$. The in vitro release study of the pure drug and ATM$2-\mathrm{HP}-\beta-\mathrm{CD}$ inclusion complex were studies according to the dialysis method. The accumulative release rate of the ATM-inclusion complex is $82 \%$ in $3 \mathrm{~min}$ and up to $99 \%$ in $60 \mathrm{~min}$, whereas the pure drug exhibited the release rate of only $18 \%$ in $30 \mathrm{~min}$ and $80 \%$ in $600 \mathrm{~min}$. The result showed that the solubility and release percentage of inclusion complex was improved increasing in contrast to the pure drug, verifying the effect of $2-\mathrm{HP}-\beta-\mathrm{CD}$ on improving the dissolution percentage of insoluble drugs. The in-vivo pharmacokinetics of ATM-2-HP- $\beta-$ CD inclusion complex was evaluated by

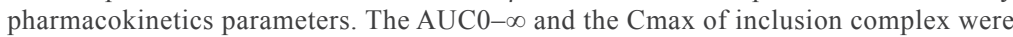
1.44-fold and 2.11-fold greater than that of ATM solution which indicated that the bio-availability of ATM has notably increased with the complexation of $2-\mathrm{HP}-\beta-\mathrm{CD}$. The tmax, $\mathrm{t} 1 / 2$ and MRT $(0-\infty)$ of inclusion complex were $0.67,0.66$ and $0.94-$ fold lower than that of ATM solution which indicated that the inclusion complex has fast blood absorption. The data analyzed above demonstrated that the bio-availability of insoluble drugs can be improved notably by inclusion technique.
\end{abstract}

Keywords: artemether, 2-hydroxypropyl- $\beta$-cyclodextrin, inclusion complex, solubility, bio-availability
Volume 3 Issue 6 - 2017

Yanyun Hao, Shilei Chen, Liu Tian, Zhenlei
Yang, Guihua Huang
Shandong University, School of Pharmaceutical Sciences, China

Correspondence: Guihua Huang, School of Pharmaceutical Sciences, Shandong University, 44 Wenhua Xi Road, Jinan, 2500 I2, Shandong, China, Tel (086)053I-883820I5, Fax (086)053I-88382548, Email hgh2003@gmail.com

Received: June 30, 2017 | Published: September 0I, 2017
Abbreviations: ATM, artemether; DSC, differential scanning calorimetry; FT-IR, fourier transform infrared spectroscopy; AUC, area under curve; MRT, mass rapid transit; CDs, cyclodextrins; HPLC, high performance liquid chromatography; LE, load efficiency

\section{Introduction}

Malaria has a serious influence on human beings especially on that in tropical and subtropical countries. It is reported that there are approximately $300-500$ million in clinical and 1.5-2.7 million in death every year. Among them, Plasmodium falciparum is the main protozoon for fatal cases. ${ }^{1}$ With the widespread drug resistance limited by older therapies dominated by quinine, standard therapy for malaria is now artemisinin-based chemotherapy which is genuine and of high quality. ${ }^{2}$ Artemether, [3R-(3R,5aS,6S, $8 \mathrm{aS}, 9 \mathrm{R}$ ,10R,12S,12aR)]-Decahydro-10-methoxy-3,6,9-trimethyl-3,12epoxy-12H-pyrano[4,3-j]-1,2-benzodioxepin, ${ }^{3}$ is one of the lipidsoluble derivative of artemisinin, which has proved to be efficient against malaria especially for malignant malaria. ${ }^{4}$ The structural formula is shown in Figure 1A. ATM disturbs the mitochondrial function, influence ultrastructure, and block the nutritional intake of
Plasmodium falciparum, making them to lose cytoplasm and nutrients and cannot get replenish and soon died. ${ }^{5,6}$

ATM belongs to BCS IV drug with poorly soluble and poorly permeable peculiarity. ${ }^{7}$ ATM is only available as a pre-mixed oilbased solution for intramuscular injection. And there is evidence that the intramuscular injection may not be absorbed absolutely and can cause terrible pain to patient. ${ }^{8,9}$ The novel delivery system such as liposome ${ }^{10}$ and nanostructured lipid ${ }^{11}$ of ATM did not achieve its aim of improving anti-malarial therapeutic efficiency. In addition, its poor bioavailability and rapid clearance from the body limits its exploitation and application in clinical study. From the analysis mentioned above, we can realize the importance of improving its solubility and bioavailability. Several formulation approach could be used to improving the solubility along with bio-availability of insoluble drugs. ${ }^{12}$ Until now, different formulations are reported such as Solid Dispersion System, ${ }^{7}$ nanoparticle, ${ }^{13}$ inclusion complex. ${ }^{1}$ Among them, inclusion complex is widely elected due to their mass production achieved easily.

As a kind of functional excipients, cyclodextrins (CDs) have been used extensively in pharmaceutical formulations to improve the bio- 
availability of insoluble drugs. ${ }^{14-17}$ Their unique physicochemical property including a hydrophilic external surface and an internal hydrophobic cavity allows the formation of host-guest interactions with hydrophobic organic compounds. ${ }^{18}$ The widely natural CDs are made up of 6-8 oligosaccharides units joined with each other through $1-4$ bonds, they are called $\alpha-, \beta-, \gamma-\mathrm{CDs}$, respectively. ${ }^{19}$ Among them, $\beta-\mathrm{CD}$ has the lower toxicity compared with the $\alpha-, \gamma-\mathrm{CDs}$ and it can fit most of the aromatic backbone of ATM because of its cavity, but the solubility of it in water is only $18.5 \mathrm{~g} / \mathrm{L}$ which hampers the absorption of drugs, Nowadays, the tendency is to modify the parent $\beta-C D$ resulting into derivatives which can overcome the disadvantages existed in $\beta-\mathrm{CD}, 2-\mathrm{HP}-\beta-\mathrm{CD}$ is one of the most suitable derivatives with high capacity of complexion, high solubility of $750 \mathrm{~g} / \mathrm{L}$ approximately and lower toxicity. The structural formula is shown in Figure $1 \mathrm{~B},{ }^{20,21}$ thus we select $2-\mathrm{HP}-\beta-\mathrm{CD}$ as the excipient of infusion complex. In order to improve the solubility, stability and bio-availability of ATM, we prepared ATM-2-HP- $\beta-\mathrm{CD}$ inclusion complex. ${ }^{22}$ The ATM-2-HP- $\beta-\mathrm{CD}$ inclusion complex obtained by Solution mixing method under the optimum conditions was characterized using DSC, FTIR, and HPLC chromatography analyses. In addition, the appearance, melting point, dissolution, solubility characteristics and pharmacokinetic parameter of the ATM-2-HP- $\beta$ CD inclusion complex were also researched in this study.

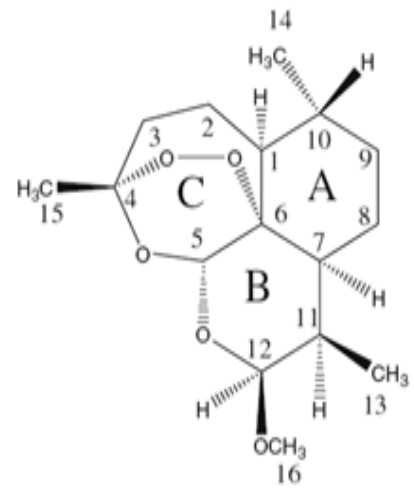

A

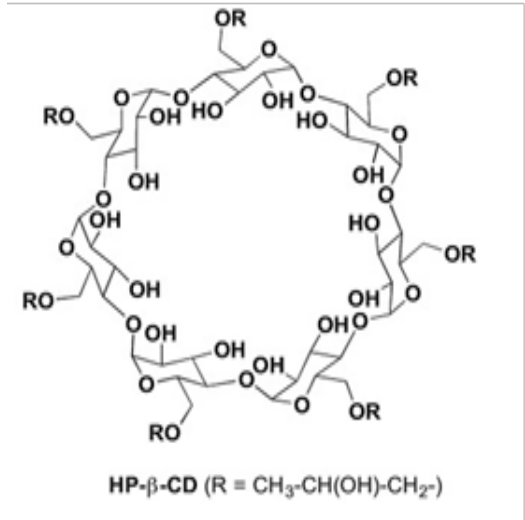

Figure I The structural formula for: (A) ATM. (B) $2-H P-\beta-C D$.

\section{Materials and methods}

\section{Materials}

ATM was purchased from Kunming pharmaceutical factory (Kunming, China). 2-HP- $\beta-\mathrm{CD}$ was purchased from Shandong
Binzhou Zhiyuan Bio-Technology Co. Ltd. (Binzhou, China), with the theory molar substitution of 4.7. Hydrochloric acid was purchased from Shandong Laiyang economic and technological development zone chemical factory (Laiyang Shandong). Absolute ethanol was purchased from Tianjin Fuyu Chemical Co. Ltd. (Tianjin, China). Acetonitrile is of grade and purchased from Tedia Company (USA). ATM $-2-\mathrm{HP}-\beta-\mathrm{CD}$ is made by ourselve. The water is distilled water. All other reagents used were of analytical grade.

\section{Methods}

Preparation method selection of ATM-2-HP- $\beta-C D$ inclusion complex: There are many methods for inclusion complex preparation including grinding, ultrasonic, solution mixing and so on. This study selects the best suitable one for ATM-2-HP- $\beta-\mathrm{CD}$ inclusion complex on the basis of inclusion rate (IR) and Load efficiency (LE) as the evaluation index.

Grinding method: ${ }^{23}$ The accurately weighed mixture of ATM and $2-$ HP- $\beta-C D(1: 40)$ was triturated in a mortar with a small volume of water- methanol $(1: 1 \mathrm{v} / \mathrm{v})$ solution for about $90 \mathrm{~min}$. The ethanol was volatilized after a period of time and then the inclusion complex was collected.

Ultrasonic wave method: The accurately weighed mixture of ATM and $2-\mathrm{HP}-\beta-\mathrm{CD}(1: 40)$ was taken into a tomato type bottle with $10 \mathrm{ml}$ ethanol and a small amount of water. After the sustained ultrasonic for $1 \mathrm{~h}$, the ethanol was volatilized with a Rotary Evaporator at $50^{\circ} \mathrm{C}$. Then, the inclusion complex was collected.

Solution mixing method: The accurately weighed mixture of ATM and $2-\mathrm{HP}-\beta-\mathrm{CD}(1: 40)$ was taken into a tomato type bottle with $10 \mathrm{ml}$ ethanol and a small amount of water. Added another little water to dissolve the ATM and $2-\mathrm{HP}-\beta-\mathrm{CD}$ by stirring $3-5 \mathrm{~min}$. constantly dripping the remaining water slowly, continue stirring for $1.5 \mathrm{~h}$ and then remove magnon. The inclusion complex was collected by $50^{\circ} \mathrm{C}$ decompression spin steaming.

Determination of IR and $L E$ of $A T M-2-H P-\beta-C D$ inclusion complex: The IR\% and LE\% of ATM-2-HP- $\beta-C D$ inclusion complex were determined indirectly by UV spectroscopy method. Briefly, a suitable amount of ATM was added in a volumetric flask $(10 \mathrm{~mL})$ and mixed with ethanol up to $10 \mathrm{~mL}$. After mixing finished, $100 \mu \mathrm{l}$ of $10 \mathrm{ml}$ solution was taken into a another volumetric flask $(10 \mathrm{~mL})$, and then reacts with hydrochloric acid $(1 \mathrm{~mol} / \mathrm{L})$ up to $10 \mathrm{~mL}$; at the same time, a suitable amount of ATM-2-HP- $\beta-$ CD inclusion complex was added in a volumetric flask $(10 \mathrm{~mL})$ and reacted with hydrochloric acid $(1 \mathrm{~mol} / \mathrm{L})$ up to $10 \mathrm{~mL}$. The solution prepared above was heated at $80 \pm 2^{\circ} \mathrm{C}$ for $20 \mathrm{~min}$ and the absorbance was measured. The IR $\%$ and LE\% of the ATM-2-HP- $\beta-$ CD inclusion complex were calculated with the equation as follows:

$$
\begin{aligned}
& L E \%=\frac{\frac{A 2}{A 1} \times \frac{m 1}{100}}{m 2} \times 100 \% \\
& I R \%=\frac{W \times m}{m} \times 100 \%
\end{aligned}
$$

In the equation (1): $\mathrm{LE} \%$ is the load efficiency of the ATM- $-\mathrm{HP}-\beta-$ $\mathrm{CD}$ inclusion complex

$A_{1}$ is the absorbance of the ATM solution after the reaction with hydrochloric acid 
$\mathrm{A}_{2}$ is the absorbance of the ATM-2-HP- $\beta-\mathrm{CD}$ inclusion complex after the reaction with hydrochloric acid

$\mathrm{m}_{1}$ is the amount of ATM weighed

$\mathrm{m}_{2}$ is amount of ATM-2-HP- $\beta-\mathrm{CD}$ inclusion complex weighed

In the equation (2): IR\% is the inclusion rate of ATM- $-\mathrm{HP}-\beta-\mathrm{CD}$ inclusion complex

$\mathrm{W}$ is the amount of the drug ATM-2-HP- $\beta-\mathrm{CD}$ inclusion complex

$\mathrm{m}$ ' is the mass of the inclusion complex

$\mathrm{m}$ is the mass of the ATM at the time of feeding

Optimization of ATM $-2-\mathrm{HP}-\boldsymbol{\beta}-\mathbf{C D}$ inclusion complex: On the basis of preliminary test, the ATM-2-HP- $\beta-\mathrm{CD}$ inclusion complex was prepared by solution mixing method. At the same time, singlefactor screening experiment was used to optimize the best formulation. There are many factors affecting Load efficiency (LE\%) and inclusion rate (IR\%) including material inputting ratio, stirring time and stirring temperature. With the consideration discussed just now, the ratio of drug and $2-\mathrm{HP}-\beta-\mathrm{CD}$ are tested at $1: 25,1: 30,1: 36$ and $1: 40$ respectively. Stirring times are tested at $1 \mathrm{~h}, 2 \mathrm{~h}$ and $3 \mathrm{~h}$ respectively. Stirring temperatures are tested at $40^{\circ} \mathrm{C}, 50^{\circ} \mathrm{C}$ and $60^{\circ} \mathrm{C}$ respectively.

\section{Characterization of ATM-2-HP- $\beta-C D$ inclusion complex}

Appearance and morphological characterization of ATM-2HP- $\boldsymbol{\beta}-\mathbf{C D}$ : The dried ATM-2-HP- $\beta-$ CD inclusion complex prepared by the optimal condition was placed in a dry penicillin bottle, and then observed its appearance by the naked eyes.

Differential scanning calorimetry (DSC): The thermal characteristics of the ATM, $2-\mathrm{HP}-\beta-\mathrm{CD}$, their physical mixture, and the ATM-2$\mathrm{HP}-\beta-\mathrm{CD}$ inclusion complex were performed using DSC. Samples above were accurately weighed and sealed in an aluminum pan. They were then placed in the instrument and heated at a rate of $15^{\circ} \mathrm{C} / \mathrm{min}$ from 40 to $350^{\circ} \mathrm{C}$ under a constant flow $(25 \mathrm{~mL} / \mathrm{min})$ of nitrogen gas. An empty sealed pan was used as a reference.

Fourier transform infrared spectroscopy (FT-IR): The FT-IR spectra of ATM, $2-\mathrm{HP}-\beta-\mathrm{CD}$, their physical mixture, and the ATM$2-\mathrm{HP}-\beta-\mathrm{CD}$ inclusion complex were obtained using FT-IR. Samples above were prepared and mixed with spectrograde $\mathrm{KBr}$ powder. The spectra produced by samples above were gained from $4000 \mathrm{~cm}^{-1}$ to $400 \mathrm{~cm}^{-1}$.

The solubility of ATM-2-HP- $\boldsymbol{\beta}-\mathbf{C D}$ inclusion complex: The solubility of ATM-2-HP- $\beta-$ CD inclusion complex was carried out according to the method of Ultraviolet spectrophotometer. The ATM$2-\mathrm{HP}-\beta-\mathrm{CD}$ inclusion complex prepared under the optimal conditions was filtered with syringe through a $0.45 \mu \mathrm{m}$ filter membrane. Taking further filtrate $100 \mathrm{uL}$ into $10 \mathrm{ml}$ volumetric flask and fixed volume with $1 \mathrm{~mol} / \mathrm{L}$ hydrochloric acid, and then react $20 \mathrm{~min}$ under the condition of $80 \pm 2^{\circ} \mathrm{C}$. Regarded ATM standard solution as the control, the amount of ATM-2-HP- $\beta-\mathrm{CD}$ inclusion complex in solution was determined by measuring the UV absorbance at $257 \mathrm{~nm}$.

The Melting point of ATM-2-HP- $\boldsymbol{\beta}-\mathbf{C D}$ inclusion complex: The Melting point of ATM-2-HP- $\beta-\mathrm{CD}$ inclusion complex was evaluated by the method of capillary. Firstly, taking a side sealing capillary insert reagent bottles filled with powder with the thickness ranged from 2 to $3 \mathrm{~mm}$ and wiping its outer wall. Secondly, throwing down the prepared side sealing capillary from the top of an air condenser pipe and repeating several times. Lastly, the welled gained capillary tube was inserted into the melting point instrument. After heating the sample to about $75^{\circ} \mathrm{C}$ rapidly, slowing down the speed of heating and observing the phenomenon of melting point and record the reading. Samples were measured three times after cooling temperature by $20^{\circ} \mathrm{C}$.

The stability of ATM-2-HP- $\boldsymbol{\beta}-\mathbf{C D}$ inclusion complex: The stability of ATM-2-HP- $\beta-$ CD inclusion complex was carried by the method offered in Chinese Pharmacopoeia (Ch. P). Taking a suitable amount of ATM in penicillin bottles whose thickness is $5 \mathrm{~mm}$ or less. High temperature $\left(50^{\circ} \mathrm{C}\right)$, high moisture $(75 \%, 92.5 \%)$, and high light $(45001 \mathrm{x}-5001 \mathrm{x})$ experiment are carried with sampling in 5-10 days respectively. The stability of ATM-2-HP- $\beta-\mathrm{CD}$ inclusion complex was testified by detecting the contents of ATM in inclusion complex.

The in-vitro release studies of ATM and ATM-2-HP- $\beta-C D$ inclusion complex: The dissolution patterns of the pure drug $(2 \mathrm{mg})$ and ATM-2-HP- $\beta-\mathrm{CD}$ inclusion complex (equivalent to $2 \mathrm{mg}$ ATM) were studies according to the dialysis method (molecular weight cutoff is $1 \mathrm{k}_{\mathrm{Da}}$. The samples were placed to a dialysis bag with $5 \mathrm{~mL}$ release media of PBS with $\mathrm{p}^{\mathrm{H}} 7.4$ and dialyzed against $25 \mathrm{~mL}$ release media at $37 \pm 0.5^{\circ} \mathrm{C}$ in a water bath shaker at $100 \mathrm{rpm}$. At scheduled time $(3,5,10,15,20,30,45,60,120.240 .360,480$ and 600h) intervals, $4 \mathrm{~mL}$ of the release media was collected and then the same volume of fresh release media was added. The withdrawn samples were filtered through a $0.45 \mu \mathrm{m}$ membrane filter. The release content of ATM was determined by UV at $254 \mathrm{~nm}$. The accumulative release percentage of $\operatorname{ATM}(\mathrm{Q} \%)$ was calculated according to the equation as followed:

$$
Q=\frac{C_{i} V+\left(C_{i-1}+C i-2 \mathcal{V}_{i-2}+\ldots \ldots . .+C_{1} \mathcal{V}_{1}\right)}{W} \times 100 \%
$$

\section{Where $\mathrm{Q}$ is the release rate}

$\mathrm{C}_{1}$ is the drug concentration in the release medium of the first time interval

$\mathrm{C}_{\mathrm{i}}$ is the drug concentration in the release medium of each time interval

$\mathrm{V}$ is the total volume of the release medium

$\mathrm{V}_{1}$ is the volume of the withdrawn medium

$\mathrm{V}_{\mathrm{i}}$ is the drug concentration in the release medium at time

$\mathrm{W}$ is the total drug content in the dialysis bag.

Pharmacokinetic study: The pharmacokinetic study was carried out on six adult Wistar female rats weighing $200 \pm 20 \mathrm{~g}$. The rats used were divided into two groups randomly. One group was given ATM suspension through intragastric administration, while the other was given ATM-HP- $\beta-$ CD inclusion complex, both with dosage of 30 $\mathrm{mg} / \mathrm{kg}$. At specified time intervals after intragastric administration $(0.25,0.5,0.75,1,2,3,4,6,8 \mathrm{~h}), 0.5 \mathrm{~mL}$ blood samples was collected from jugular venous sinus. Then the $200 \mu$ l of blood samples were put into $1.5 \mathrm{~mL}$ of centrifugal Tubes washed with heparin sodium. $300 \mu \mathrm{l}$ mobile phase was added and the mixture was vortexed for $3 \mathrm{~min}$. Next, the mixture gained above was centrifuged for $10 \mathrm{~min}$ under the condition of $12,000 \mathrm{rpm}$, and then filter the supernatant through a $0.22 \mu \mathrm{m}$ filter membrane. Finally, $20 \mu \mathrm{l}$ of the filtered solution was injected into the HPLC instrument and the concentration was analyzed by the HPLC method mentioned below. The concentration of ATM 
in plasma of rat was calculated according to the standard curve of " $\mathrm{A}=866.31 \mathrm{C}+1807.2, \quad \mathrm{r}=0.9994$ "and pharmacokinetic parameters were collected by DAS 2.0 software (Table 1 ).

Table I Pharmacokinetic parameters were collected by DAS 2.0 software

\begin{tabular}{ll}
\hline Conditions & Parameter \\
\hline HPLC Equipment & $\begin{array}{l}\text { Shimadzu LC-10A system (Shimadzu, } \\
\text { Kyoto, Japan) }\end{array}$ \\
$\begin{array}{l}\text { Chromatography } \\
\text { Column }\end{array}$ & $\begin{array}{l}\text { Platisil TM-ODS HPLC Column with the } \\
\text { Size of } 4.6 \times 250 \mathrm{~mm}\end{array}$ \\
Mobile Phase & acetonitrile-Deionized water $(77: 23 \mathrm{v} / \mathrm{v})$ \\
Flow Rate & $\begin{array}{l}1.0 \mathrm{ml} / \mathrm{min} \\
\text { Detection Wavelength } \\
\text { Column Temperature }\end{array}$ \\
$\begin{array}{l}210 \mathrm{~nm} \\
\text { Sample Volume }\end{array}$ & $20 \mu \mathrm{L}$ \\
\hline
\end{tabular}

\section{Results and discussion}

\section{Preparation method selection of ATM-2-HP- $\beta-C D$ inclusion complex}

Grinding method for the preparation of ATM-2-HP- $\beta-C D$ inclusion complex is the worst due to the little drugs was complexed into the $2-\mathrm{HP}-\beta-\mathrm{CD}$. This result can be gained from the floating objects on the surface of the solution. In addition, this method is laborious and exposed to the air. Ultrasonic wave and solution mixing method are suitable for the preparation of inclusion compound without floating objects, but the former can cause damage to the instrument due to its long sustained time for preparation. On the contrary, solution mixing has low requirement to the instrument, easy to operate, becoming the most suitable method for the preparation of ATM-2-HP- $\beta-$ CD inclusion complex.

\section{Optimization of ATM-2-HP- $\beta-C D$ inclusion complex}

Single-factor including the ratio, stirring time and stirring temperature was critical for preparation of the inclusion complex of ATM $-2-\mathrm{HP}-\beta-\mathrm{CD}$. Load efficiency and inclusion rate were mainly affected by the ratio of drug and $2-\mathrm{HP}-\beta-\mathrm{CD}$, not the stirring time and stirring temperature. Thus, the optimal condition of each factor was $1: 36,1 \mathrm{~h}$ and $50^{\circ} \mathrm{C}$ respectively. The reproducibility test of ATM$2-\mathrm{HP}-\beta-\mathrm{CD}$ inclusion complex by the conditions above verified the stability of the preparation also. The details are showed in the following figure called Figures $2 \& 3$ and Table 2.

Table 2 Three reproducibility test of ATM-2-HP- $\beta-C D$ inclusion complex

\begin{tabular}{lll}
\hline Batches & LE\% & EE\% \\
\hline 1 & $2.65 \%$ & $98.15 \%$ \\
2 & $2.67 \%$ & $98.89 \%$ \\
3 & $2.61 \%$ & $96.67 \%$ \\
Average \pm SD & $2.64 \pm 0.02 \%$ & $97.90 \pm 0.92 \%$ \\
\hline
\end{tabular}

\section{Verification of ATM-2-HP- $\beta-C D$ inclusion complex}

Appearance and morphological characterization of ATM-2HP- $\boldsymbol{\beta}-\mathbf{C D}$ : The freeze-dried powder of ATM $-2-\mathrm{HP}-\beta-\mathrm{CD}$ inclusion complex was shown in Figure 4A \& 4B. The powder was white and smooth. It is also soluble in distilled water.
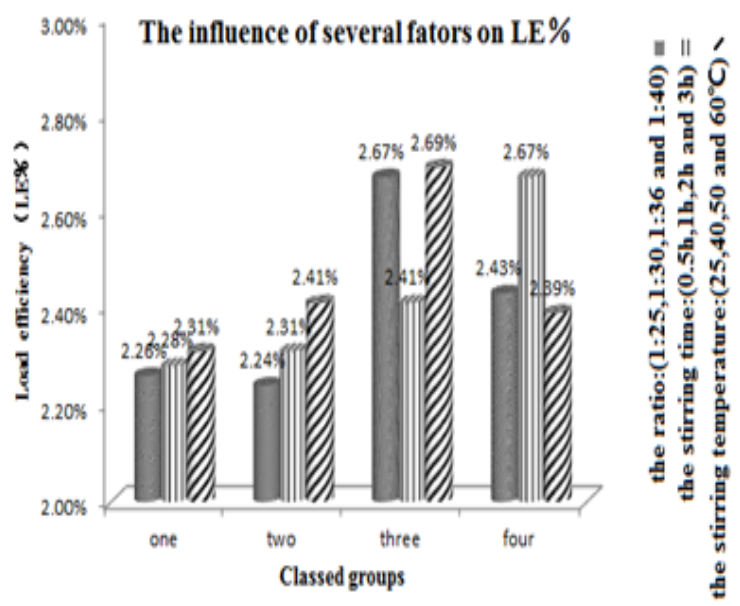

Figure $\mathbf{2}$ Load efficiency (LE) affected by the ratio of ATM and $2-H P-\beta-C D$, stirring time and stirring temperature respectively.

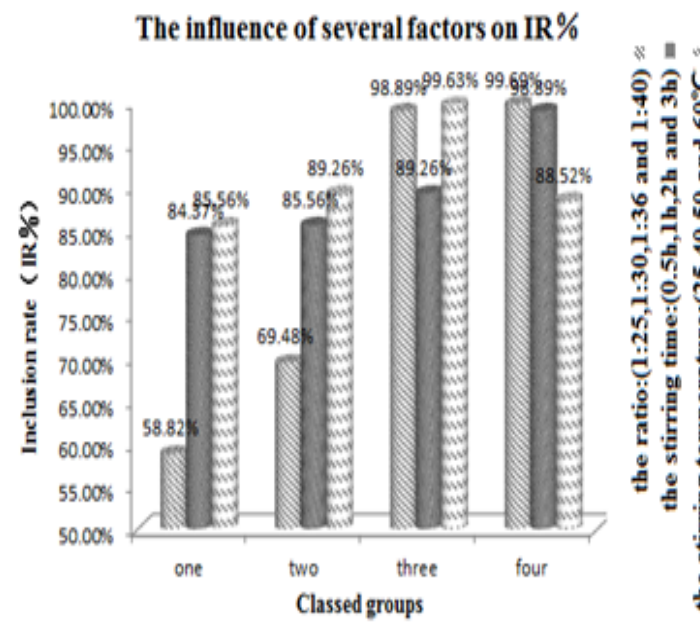

Figure 3 Inclusion rate (IR) affected by the ratio of ATM and $2-H P-\beta-C D$, stirring
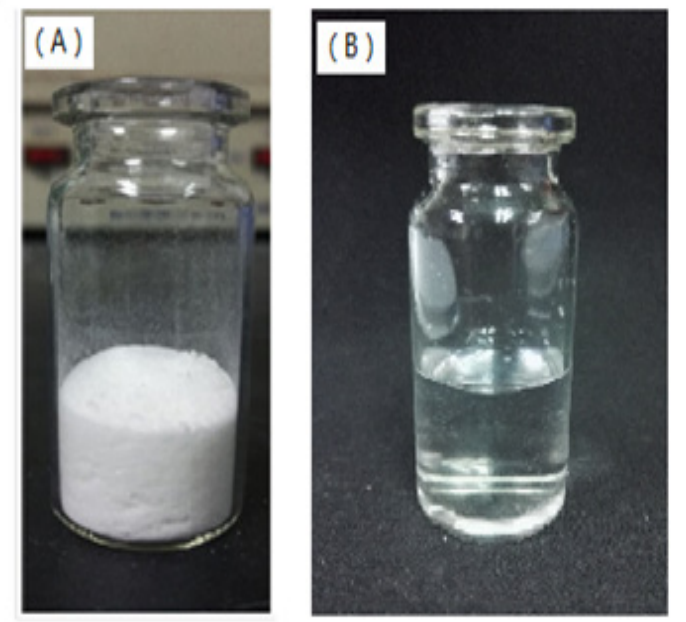

Figure 4 The appearance for: (A) ATM-2-HP- $\beta-C D$ freeze-dried powder. (B) ATM-2-HP- $\beta-C D$ aqueous solution 
Differential scanning calorimetry (DSC): The DSC curves of ATM, $2-\mathrm{HP}-\beta-\mathrm{CD}$, their physical mixture, ATM-2-HP- $\beta-\mathrm{CD}$ inclusion complex were showed in Figure 5. ATM has a typically sharp endothermic peak at $88^{\circ} \mathrm{C}$ corresponds to the melting point of ATM $\left(86-88^{\circ} \mathrm{C}\right)$; There has been a sharp exothermic peak at $174^{\circ} \mathrm{C}$, showing that the decomposition of ATM happened here. While $2-\mathrm{HP}-\beta-\mathrm{CD}$ has a large characteristic absorption peak at about $310^{\circ} \mathrm{C}$. Different from the absorption peak showed above, the physical mixture is the superposition of the both. The absorption peak at $88^{\circ} \mathrm{C}$ (ATM) and $174^{\circ} \mathrm{C}(2-\mathrm{HP}-\beta-\mathrm{CD})$ disappeared in the ATM-2-HP- $\beta-\mathrm{CD}$ inclusion complex verified the formulation of new compound.

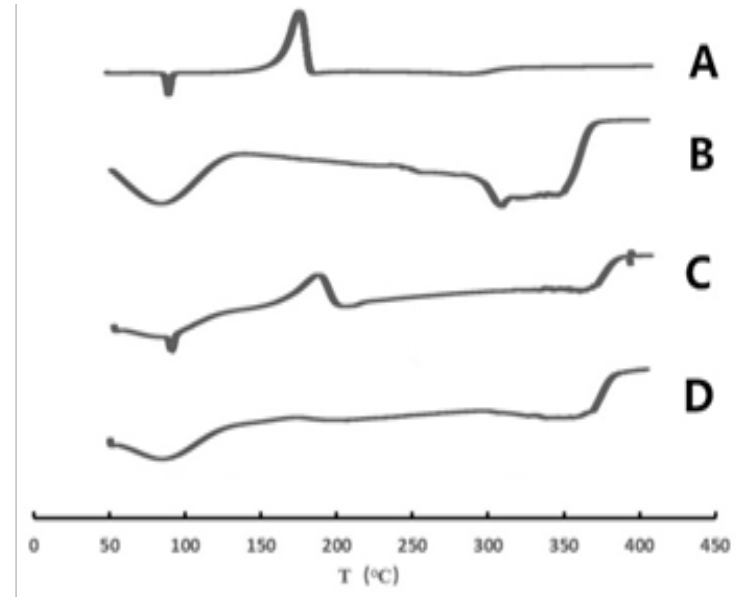

Figure 5 DSC curves for (A) ATM. (B) 2-HP- $\beta-C D$. (C) Their physical mixture. (D) The ATM-2-HP- $\beta-C D$ inclusion complex.

Fourier transform infrared spectroscopy (FT-IR): The FT-IR spectra of ATM, $2-\mathrm{HP}-\beta-\mathrm{CD}$, their physical mixture, ATM-2-HP- $\beta-$ $\mathrm{CD}$ inclusion complex were showed in Figure 6. The absorption bands of these substances are analyzed as follows:

ATM: C-H stretching vibration (2953.60, 2915.78, 2873.39, $\left.2915.78 \mathrm{~cm}^{-1}\right)$, O-H curve vibration $\left(1451.70 \mathrm{~cm}^{-1}\right), \mathrm{C}-\mathrm{H}$ curve vibration $\left(1384.37,1374.95 \mathrm{~cm}^{-1}\right), \mathrm{C}-\mathrm{O}$ stretching vibration $(1251.70$, $\left.1207.88,1189.38 \mathrm{~cm}^{-1}\right)$ etc.

2-HP- $\boldsymbol{\beta}-\mathbf{C D}$ : O-H curve vibration $\left(3405.98 \mathrm{~cm}^{-1}\right), \mathrm{H}-\mathrm{O}-\mathrm{H}$ curve vibration $\left(1645.70 \mathrm{~cm}^{-1}\right), \mathrm{C}-\mathrm{H}$ curve vibration $\left(2926.05 \mathrm{~cm}^{-1}\right)$.

The spectrum of the physical mixture was evidently the superposition of the absorption spectra of ATM and $2-\mathrm{HP}-\beta-\mathrm{C}$

The spectrum of the ATM-2-HP- $\beta-\mathrm{CD}$ inclusion complex has no absorption band of $\mathrm{C}-\mathrm{O}$ stretching vibration (1251.70, 1207.88, $1189.38 \mathrm{~cm}^{-1}$ ) compared with that of ATM. The analysis verified that the ATM has been entrapped in the cavity of $2-\mathrm{HP}-\beta-\mathrm{CD}$.

The determination of solubility: The solubility experiment showed that the solubility in water of ATM-2-HP- $\beta-$ CD inclusion complex was $11.28 \pm 2.26 \mathrm{mg} / \mathrm{ml}$, having improved significantly with the ATM of just $1.18 \mathrm{ug} / \mathrm{ml} .^{24}$

The determination of melting point: The melting point reported of pure ATM and $2-\mathrm{HP}-\beta-\mathrm{CD}$ is $86-89^{\circ} \mathrm{C}, 278^{\circ} \mathrm{C}$, respectively. $226-229^{\circ} \mathrm{C}$ were seen for the ATM-2-HP- $\beta-\mathrm{CD}$ inclusion complex in experiment, which indicating that the formulation of inclusion complex.
The evaluation of stability: The result of stability of ATM- $-2-\mathrm{HP}-\beta-$ $\mathrm{CD}$ inclusion complex was shown in Table 3 . The experiment showed that the ATM-2-HP- $\beta-$ CD inclusion complex is not stable in high temperature and moisture conditions but stable in illumination. Thus, it should be preserved in dry at low temperature.
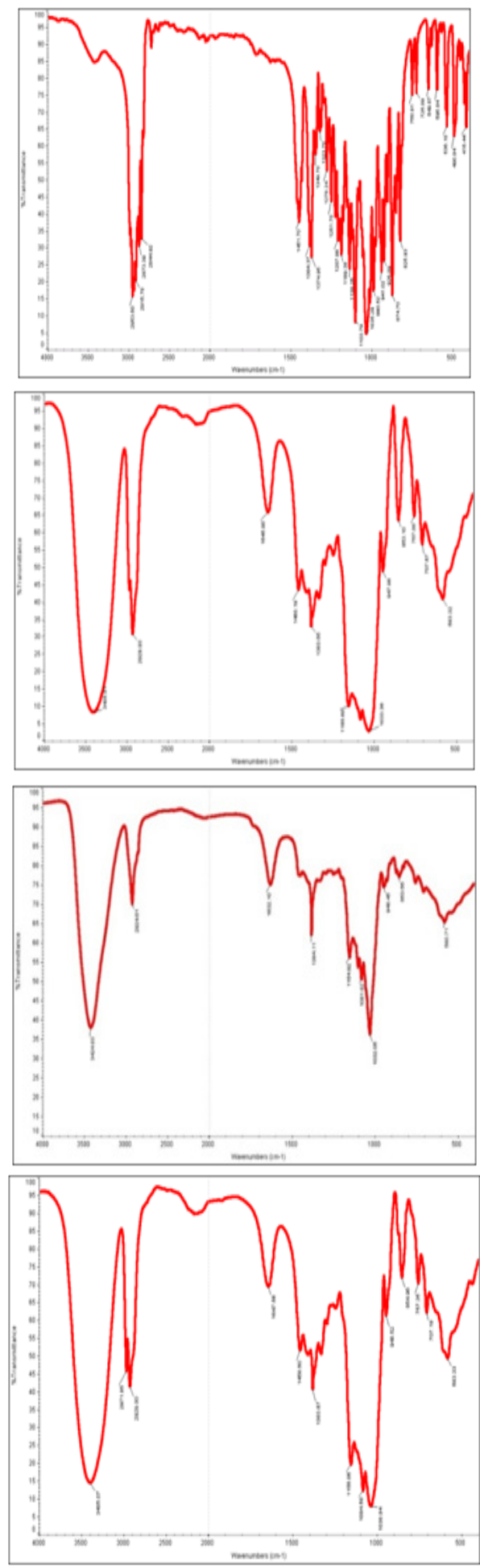

Figure 6 The FT-IR spectra for: (A) ATM. (B) 2-HP- $\beta-C D$. (C) Their physical mixture. (D) The ATM-2-HP- $\beta-C D$ inclusion complex. 
The in vitro studies of ATM and ATM-2-HP- $\beta-C D$ inclusion complex: The release behavior profile was drawn as the cumulative release rate (\%) on the $\mathrm{y}$-axis and time $(\mathrm{min})$ on the $\mathrm{x}$-axis shown in Figure 7. It showed that the accumulative release rate of the ATMinclusion complex is $82 \%$ in $3 \mathrm{~min}$ and up to $99 \%$ in $60 \mathrm{~min}$, whereas the pure drug exhibited the release rate of only $18 \%$ in $30 \mathrm{~min}$ and $80 \%$ in $600 \mathrm{~min}$. It was evident that the inclusion complex exhibited the faster dissolution rate than ATM alone. This result verified the effect of $2-\mathrm{HP}-\beta-\mathrm{CD}$ on improving the dissolution efficiency of insoluble drugs.

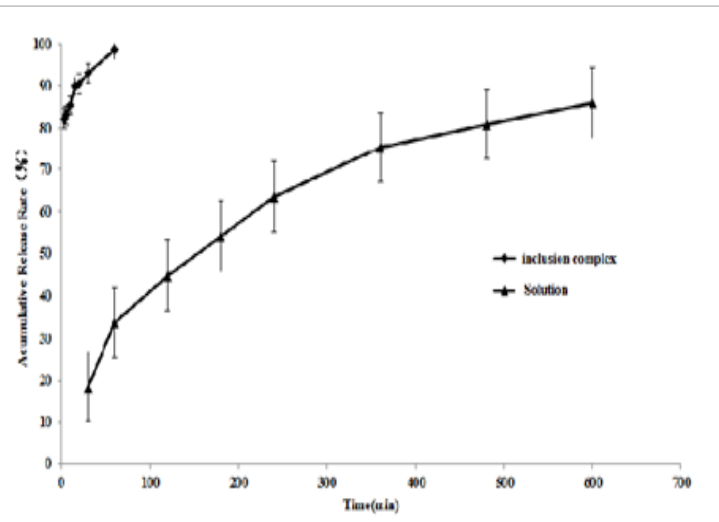

Figure 7 The in-vitro dissolution studies of ATM-2-HP- $\beta-C D$ inclusion complex and ATM.

Pharmacokinetics and bioavailability study: The plasma concentration versus time profiles of ATM-2-HP- $\beta-$ CD inclusion complex and ATM were shown in Figure 8. The results of pharmacokinetic parameters were shown in Table 4 . The $t_{1 / 2}(h)$ of the ATM-2-HP- $\beta-$ CD inclusion complex is 0.66 times lower to that of

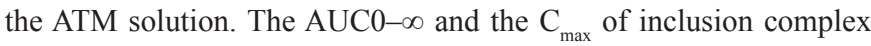
of ATM were 1.44 times and 2.11 times greater than that of ATM solution which indicated that the bio-availability of ATM has notably increased with the complexation of $2-\mathrm{HP}-\beta-\mathrm{CD}$. The $\mathrm{t}_{\text {max }}$ and MRT $(0-\infty)$ of inclusion complex were 1.51 times and 1.06 times lower than that of ATM solution which indicated that the inclusion complex has fast blood absorption.

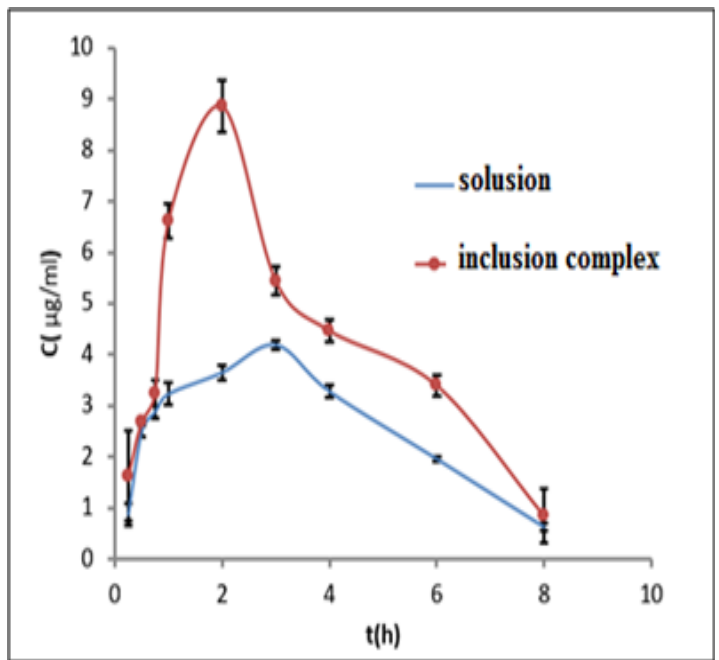

Figure 8 The plasma concentration versus time profiles for: (A) ATM. (B) $2-\mathrm{HP}-\beta-\mathrm{CD}$
Pharmacokinetics and bioavailability study: The plasma concentration versus time profiles of ATM-2-HP- $\beta-$ CD inclusion complex and ATM were shown in Figure 8. The results of pharmacokinetic parameters were shown in Table 3 . The $t_{1 / 2}(h)$ of the ATM-2-HP- $\beta-$ CD inclusion complex is 0.66 times lower to that of the ATM solution. The AUC $0-\infty$ and the $\mathrm{C}_{\max }$ of inclusion complex of ATM were 1.44 times and 2.11 times greater than that of ATM solution which indicated that the bio-availability of ATM has notably increased with the complexation of $2-\mathrm{HP}-\beta-\mathrm{CD}$. The $t$ max and MRT $(0-\infty)$ of inclusion complex were 1.51 times and 1.06 times lower than that of ATM solution which indicated that the inclusion complex has fast blood absorption.

Table 3 Stability of ATM-2-HP- $\beta-C D$ inclusion complex

\begin{tabular}{|c|c|c|c|c|}
\hline \multirow{2}{*}{ Condition } & \multicolumn{2}{|l|}{5 Days } & \multicolumn{2}{|l|}{10 Days } \\
\hline & Appearance & Content & Appearance & Content \\
\hline $\begin{array}{l}\text { High } \\
\text { Temperature }\end{array}$ & $\begin{array}{l}\text { White } \\
\text { Granules }\end{array}$ & $2.61 \%$ & $\begin{array}{l}\text { White } \\
\text { Granules }\end{array}$ & $2.59 \%$ \\
\hline RH 75\% & Agglomeration & $2.43 \%$ & Agglomeration & $2.35 \%$ \\
\hline RH 92.5\% & $\begin{array}{l}\text { Transparent } \\
\text { Gel }\end{array}$ & --- & $\begin{array}{l}\text { Transparent } \\
\text { Gel }\end{array}$ & --- \\
\hline Illumination & $\begin{array}{l}\text { White Fine } \\
\text { Powder }\end{array}$ & $2.63 \%$ & $\begin{array}{l}\text { White Fine } \\
\text { Powder }\end{array}$ & $2.61 \%$ \\
\hline
\end{tabular}

Table 4 Main pharmacokinetic parameters of ATM and ATM-2-HP- $\beta-C D$ inclusion complex

\begin{tabular}{lll}
\hline & ATM solution & ATM-2-HP- $\beta$-CD \\
\hline $\mathrm{t}_{1} / 2 \beta(\mathrm{h})$ & 2.73 & 1.803 \\
$\mathrm{MRT}(0-\infty)(\mathrm{h})$ & 3.94 & 3.718 \\
$\mathrm{~T}_{\text {max }}(\mathrm{h})$ & 3 & 2 \\
$\mathrm{C}_{\text {max }}(\mu \mathrm{g} / \mathrm{L})$ & 4.19 & 8.86 \\
$\mathrm{AUC} \mathrm{C}_{-\infty}\left(\mu \mathrm{g} / \mathrm{L}^{*} \mathrm{~h}\right)$ & 25.55 & 36.88 \\
\hline
\end{tabular}

\section{Conclusion}

In this study, the ATM-2-HP- $\beta-\mathrm{CD}$ inclusion complex was successfully prepared through Solution mixing method at the mole ratio of 1:36 (ATM: $2-\mathrm{HP}-\beta-\mathrm{CD}$ ). The formulation of inclusion complex was verified by DSC and FT-IR. The solubility of ATM-2HP- $\beta-C D$ was $11.28 \pm 2.26 \mathrm{mg} / \mathrm{ml}$, having improved vastly compared to ATM of just $1.18 \mathrm{ug} / \mathrm{ml}$. The in-intro release study of the pure drug and ATM-2-HP- $\beta-$ CD inclusion complex were studies according to the dialysis method. The accumulative release rate of the ATMinclusion complex is $82 \%$ in $3 \mathrm{~min}$ and up to $99 \%$ in $60 \mathrm{~min}$, whereas the pure drug exhibited the release rate of only $18 \%$ in $30 \mathrm{~min}$ and $80 \%$ in $600 \mathrm{~min}$. The result showed that the solubility and release percentage of inclusion complex was improved increasing in contrast to the pure drug, verifying the effect of $2-\mathrm{HP}-\beta-\mathrm{CD}$ on improving the dissolution percentage of insoluble drugs. The plasma concentration versus time profiles and pharmacokinetics study showed that the bioavailability and the absorption rate of ATM were notably increased by complexing it with $2-\mathrm{HP}-\beta-\mathrm{CD}$. 


\section{Acknowledgements}

The authors wish to give thanks to the School of Pharmaceutical Science, Shandong University, for providing helps for this study.

\section{Conflict of interest}

The author declares no conflict of interest.

\section{References}

1. Yang B, Lin J, Chen Y, et al. Artemether/hydroxypropyl- $\beta$-cyclodextrin host-guest system: characterization, phase-solubility and inclusion mode. Bioorg Med Chem. 2009;17(17):6311-6317.

2. Gemma S, Travagli V, Savini L, et al. Malaria chemotherapy: recent advances in drug development. Recent Pat Antiinfect Drug Discov. 2010;5(3):195-225.

3. Ko Hsueh Tung Pao. (3,5a,6,8a,9,10,12,12a)-Decahydro-10-methoxy-3,6,9-trimethyl-3,12-epoxy-12pyrano[4,3-]-1,2-benzodioxepin.

4. Shah PP, Mashru RC. Development and evaluation of artemether taste masked rapid disintegrating tablets with improved dissolution using solid dispersion technique. AAPS PharmSciTech. 2008;9(2):494-500.

5. Yeka A, Kigozi R, Conrad MD, et al. Artesunate/Amodiaquine Versus Artemether/Lumefantrine for the Treatment of Uncomplicated Malaria in Uganda: A Randomized Trial. J Infect Dis. 2015;213(7):1134-1142.

6. Prah J, Ameyaw EO, Afoakwah R, et al. Quality Assessment of Artemether-Lumefantrine Samples and Artemether Injections Sold in the Cape Coast Metropolis. J Trop Med. 2016;8602619.

7. Fule RA, Meer TS, Sav AR, et al. Artemether-Soluplus Hot-Melt Extrudate Solid Dispersion Systems for Solubility and Dissolution Rate Enhancement with Amorphous State Characteristics. J Pharm (Cairo). 2013:151432

8. Hien TT, Davis TM, Chuong LV, et al. Comparative pharmacokinetics of intramuscular artesunate and artemether in patients with severe falciparum malaria. Antimicrob Agents Chemother. 2004;48(11):4234-4239.

9. Tayade NG, Nagarsenker MS. Development and evaluation of artemether parenteral microemulsion. Indian J Pharm Sci. 2010;72(5):637640.

10. Chimanuka B, Gabriëls M, Detaevernier MR, et al. Preparation of betaartemether liposomes, their HPLC-UV evaluation and relevance for clearing recrudescent parasitaemia in Plasmodium chabaudi malariainfected mice. J Pharm Biomed Anal. 2002;28(1):13-22.

11. Joshi M, Pathak S, Sharma S, et al. Design and in vivo pharmacodynamic evaluation of nanostructured lipid carriers for parenteral delivery of artemether: Nanoject. Int J Pharm. 2008;364(1):119-126.
12. Liao Y, Zhang X, Li C, et al. Inclusion complex of tamibarotene with hydroxypropyl- $\beta$-cyclodextrin: Preparation, characterization, in-vitro and in-vivo evaluation. Asian Journal of Pharmaceutical Sciences. 2017;12(2):187-192.

13. Woranuch S, Yoksan R. Preparation, characterization and antioxidant property of water-soluble ferulic acid grafted chitosan. Carbohydr Polym. 2013;96(2):495-502.

14. Davis ME, Brewster ME. Cyclodextrin-based pharmaceutics:past, present and future. Nat Rev Drug Discov. 2004;3(12):1023-1035.

15. Brewster ME, Loftsson T. Cyclodextrins as pharmaceutical solubilizers. Adv Drug Deliv Rev. 2007;59(7):645-666.

16. Li J, Qihua Jiang, Ping Deng, et al. The formation of a host-guest inclusion complex system between beta-cyclodextrin and baicalin and its dissolution characteristics. J Pharm Pharmacol. 2017;69(6):663-674.

17. Carrier RL, Miller LA, Ahmed I. The utility of cyclodextrins for enhancing oral bioavailability. J Control Release. 2007;123(2):78-99.

18. Michalska P, Aneta Wojnicz, Ana Ruiz Nuño, et al. Inclusion complex of ITH12674 with 2-hydroxypropyl-beta-cyclodextrin: Preparation, physical characterization and pharmacological effect. Carbohydr Polym. 2017;157:94-104.

19. Ai F, Ma Y, Wang J, et al. Preparation, Physicochemical Characterization and In-vitro Dissolution Studies of Diosmin-cyclodextrin Inclusion Complexes. Iran J Pharm Res. 2014;13(4):1115-1123.

20. Zoeller T, Dressman JB, Klein S. Application of a ternary HP- $\beta$ $\mathrm{CD}$-complex approach to improve the dissolution performance of a poorly soluble weak acid under biorelevant conditions. Int J Pharm. 2012;430(1-2):176-183.

21. Srivalli KM, Mishra B. Improved Aqueous Solubility and Antihypercholesterolemic Activity of Ezetimibe on Formulating with Hydroxypropyl- $\beta-$ Cyclodextrin and Hydrophilic Auxiliary Substances. AAPS PharmSciTech. 2016;17(2):272-283.

22. Huang Y, Zu Y, Zhao X, et al. Preparation of inclusion complex of apigenin-hydroxypropyl- $\beta$-cyclodextrin by using supercritical antisolvent process for dissolution and bioavailability enhancement. Int J Pharm. 2016;511(2):921-930

23. Mahajan HS, SK Shah, SJ Surana. Nasal in situ gel containing hydroxy propyl $\beta$-cyclodextrin inclusion complex of artemether:development and in vitro evaluation. Journal of Inclusion Phenomena and Macrocyclic Chemistry. 2010;70(1-2):49-58.

24. Fule R, Amin P. Development and evaluation of lafutidine solid dispersion via hot melt extrusion:Investigating drug-polymer miscibility with advanced characterisation. Asian Journal of Pharmaceutical Sciences. 2014;9(2):92-106. 